\title{
Response time and response time variability as indicators of response quality during static automated perimetry
}

\author{
Judith Ungewiss ${ }^{1}$ (D) Hanspeter A. Mallot ${ }^{2} \cdot$ Ulrich Schiefer $^{1,3}$ D \\ Received: 17 December 2020 / Revised: 20 May 2021 / Accepted: 27 July 2021 / Published online: 13 September 2021 \\ (c) The Author(s) 2021
}

\begin{abstract}
Purpose Perimetry is a both demanding and strenuous examination method that is often accompanied by signs of fatigue, leading to false responses and thus incorrect results. Therefore, it is essential to monitor the response quality. The purpose of this study was to evaluate the response time (RT) and its variability (RTV) as quality indicators during static automated perimetry.

Methods Size III Goldmann stimuli (25.7') were shown with the OCTOPUS 900 perimeter in four visual field locations with 13 different stimulus luminance levels $\left(0.04-160 \mathrm{~cd} / \mathrm{m}^{2}\right)$. An increased rate of false-positive and false-negative catch trials (25\% each) served to monitor the response quality simultaneously together with response time recording. Data evaluation was divided into global and individual analysis. For global analysis, the agreement indices (AI, agreement between time periods with an increased number of false responses to catch trials and time periods with pathological response to time-based values set into relation to time periods in which only one of the two criteria was considered pathological) and for individual analysis, the Spearman correlation coefficients were calculated. Ophthalmologically normal subjects with a visual acuity $\geq 0.8$, and a maximum spherical/cylindrical ametropia of $\pm 8.00 / 2.50 \mathrm{dpt}$ were included.

Results Forty-eight subjects (18 males, 30 females, age 22-78 years) were examined. The total number of false responses to catch trials was (median/maximum): 6/82. RT and RTV were compared to the occurrence of incorrect responses to catch trials. The resulting individual Spearman correlation coefficients (median/maximum) were for RT: $\rho_{\mathrm{RT}}=0.05 / 0.35$ and for RTV: $\rho_{\mathrm{RTV}}=0.27 / 0.61$. The global analysis of the RTV showed agreement indices (median/maximum) of $\mathrm{AI}_{\mathrm{RTV}}=0.14 / 0.47$.
\end{abstract}

Conclusions According to this study, an increased portion of catch trials is suitable as a verification tool for possible response quality indicators. The RTV is a promising parameter for indicating the response quality.

Keywords Perimetry $\cdot$ Response quality $\cdot$ Response time $\cdot$ Response time variability $\cdot$ Catch trials

Judith Ungewiss

judith.ungewiss@hs-aalen.de

1 Competence Center Vision Research, Study Course Ophthalmic Optics, Aalen University of Applied Sciences, Anton-Huber-Str. 23, 73430 Aalen, Germany

2 Institute of Neurobiology, Department of Biology, Tuebingen University, Tuebingen, Germany

3 Department of Ophthalmology, Tuebingen University, Tuebingen, Germany 


\section{Key messages}

\section{What is known}

- Perimetry is monotonous and often leads to an early decrease of vigilance in patients, resulting in false responses and potentially incorrect perimetric results. Patient's responses are at the current time monitored by catch trials with a rate of 3-5\% which only allows for an overall-decision about response quality in general after the examination.

\section{What this paper adds}

- An increased number of catch trials was proven as a sufficient validation tool for the assessment of false responses and therefore vigilance during static perimetry.

- As increased catch trial rates cannot be used in clinical practice due to time constraints, false responses to catch trials were correlated to response time (RT) and response time variability (RTV) in order to indicate the quality of responses. Specifically the RTV turned out as a promising parameter.

- In the future, this method could be used to continuously monitor response quality during a perimetric session with a sufficient temporal resolution in order to terminate a perimetric session when necessary.

\section{Introduction}

Perimetry is a strenuous examination method that often leads to an increasing lack of concentration and accompanying fatigue in patients, resulting in false responses and potentially incorrect perimetric results. It is therefore advisable to monitor the quality of patient's responses continuously during perimetry.

The visual field is the entirety of visual sensory impressions that can be perceived when looking straight ahead without head and body movements [1]. The examination of the visual field within a cupola is called perimetry.

Continuous quality assessment during perimetry was first introduced by Heijl and Krakau via fixation monitoring [2, 3].

The quality of visual field examinations is nowadays usually recorded by the stability of a central fixation in combination with the number of false responses to so-called catch trials [4-6]. Due to time constraints, under clinical conditions usually a rate of only $3-5 \%$ of all stimuli is implemented as catch trials. A distinction is made between false-negative catch trials (those with highly supra-threshold, i.e. very high luminance levels which the patient would have to perceive at the corresponding normal visual field location) and falsepositive catch trials (exclusive auditory stimuli without any visual stimulus presentation at that time).

The difficulty in assessing the quality of a visual field is that differential light sensitivity (DLS) fluctuates strongly: It is usually quite high at the beginning of a visual field examination but decreases during the course of a visual field examination due to decreasing vigilance $[6,7]$.

The term vigilance is frequently used synonymously with the term "alertness" and describes the degree of central nervous activation $[8,9]$. This is subject to daytime variations and is usually higher in the morning and afternoon than at night [10]. Reduced vigilance is known daytime sleepiness [11]. In contrast to this, the term fatigue characterizes a lack of willingness to take up or sustain activities [12]. The fatigue status cannot be measured objectively, but only assessed subjectively [13]. In perimetry, fatigue is usually referred to as a decrease in visual sensitivity caused by the duration of a test procedure $[14,15]$.

Reaction time depends on the stimulus luminance level and is shorter for bright than for dim stimuli. This applies for normal as well as for defected areas $[16,17]$.

It has been reported by Surwillo and Quilter that reaction time is directly correlated to the vigilance level. In their experiment, vigilance was quantified by the percentage of pop-out stimuli being detected correctly in a visual attention-based experiment [18]. To the authors' knowledge, there are currently no studies on the correlation between vigilance, response quality and response time and its variability during perimetry.

The aim of this study is therefore on the one hand to present a method with the help of which fatigue can be generated in a standardized, dosed form and its effect can be recorded and on the other hand to evaluate the response time (RT) and response time variability (RTV) as indicators of response quality during static automated perimetry.

\section{Study design and methodology}

\section{Experimental setup}

The method of constant stimuli (MoCS), where A defined number of stimuli per defined luminance level is shown 
at each pre-defined location in randomized order [19] was used to determine DLS with the OCTOPUS 900 perimeter (Haag-Streit AG, Koeniz, Switzerland). OPI (Open Perimetry Interface [20]) was used to implement the test algorithms: The stimulus luminance was varied in 13 equal steps between $0.04(39 \mathrm{~dB})$ and $160 \mathrm{~cd} / \mathrm{m}^{2}(3 \mathrm{~dB})$ with a background luminance of $10 \mathrm{~cd} / \mathrm{m}^{2}$. Goldmann stimuli size III $\left(25.7^{\prime}\right)$ were presented 20 times each at three locations $\left(-6.1^{\circ},-3.5^{\circ}\right),\left(0^{\circ}, 7^{\circ}\right),\left(6.1^{\circ},-3.5^{\circ}\right)$ and twice each at a reference location $\left(0^{\circ}, 0^{\circ}\right)$ as an additional fixation stimulus for every luminance step. The examination included a total of 1612 stimuli within a time period of about $45 \mathrm{~min}$. Stimuli were presented for $200 \mathrm{~ms}$ with an interstimulus interval of $1500 \mathrm{~ms}$. The reaction time for each stimulus was monitored. Reaction time was defined as the time period between the presentation of a stimulus and response of the patient given by pressing the response button. An increased rate of false-positive and false-negative catch trials was interspersed (25\% each in contrast to a total share of 3-5\% under conventional clinical conditions). False-positive catch trials were defined as $0 \mathrm{~dB}$ stimuli $\left(320 \mathrm{~cd} / \mathrm{m}^{2}\right)$ and false-negative catch trials were defined as $40 \mathrm{~dB}$ stimuli $\left(0.032 \mathrm{~cd} / \mathrm{m}^{2}\right)$.

\section{Subject sample}

Forty-eight subjects, equally distributed among three age groups (21-40 years, 41-60 years, 61-80 years) were included in the study. Twenty-four dominant and 24 nondominant eyes were randomly selected. The handedness of the subjects was not evaluated. They were allowed to take the hand they preferred for operating the response button and to change hands as often as desired.

Inclusion criteria were:

- Minimum distant visual acuity (with or without correction) $\geq 0.8$ (single optotypes [numbers], VISUCAT, argus individuell optic GmbH, Ottobrunn, Germany)

- Maximum spherical/cylindrical ametropia of $\pm 8.00 / 2.50$ $\mathrm{dpt}$

- Normal ophthalmological and general health status (determined by means of an ophthalmological examination and medical history - for more information in terms of the medical history sheet and the standard examination diagnosis sheet see Online Resource 1 and Online Resource 2)

- Signed informed consent of all tested subjects

The recruitment process was as follows:

- 54 subjects of who had completed another study earlier were addressed. 23 of them agreed to take part in the present study.
- 28 more subjects (mostly employees of the Aalen University of Applied Sciences) were recruited directly for the purpose of this study, all of whom agreed to take part.

- Three subjects had to be excluded (see "Results" chapter).

The study was approved by the Ethics Committee of the State Medical Association of Baden-Wuerttemberg and all volunteers were insured during their presence and travel to and from their home town.

\section{Data evaluation}

Data evaluation was conducted using MatLab (Release 2018a, The MathWorks Inc., Natick, USA).

As the included parameters did not generally show a Gaussian distribution (tested with Shapiro-Wilk test, see "Results"), parameter-free statistical tests were selected for evaluation.

All results for the response time (RT), the response time variability (RTV) and the error rate for the individual analysis (see below) were normalized to the value range $[0 ; 1]$ prior to further evaluation.

False-positive and false-negative responses to catch trials were evaluated together for further evaluation. By definition, an increased number of false responses to catch trials was assumed if more than two errors per minute (corresponding to the 95th percentile of the error rate for all test persons) occurred.

For response time (RT) and response time variability (RTV) a linear interpolation was performed for those time phases, where no response time values were available due to the presentation of infra-threshold stimuli and correspondingly absent responses. The RTV was calculated as variance of the RT over a 60s- "sliding window" (for a simplified graphical illustration of the "sliding window" technique, see Fig. 1d).

First a global analysis (using the same criteria for all subjects) was performed, followed by an individual analysis (using individual criteria for each subject separately).

For the global analysis, sensitivity and specificity for RT and RTV were each calculated for the whole group of subjects, which means, that all RT and RTV values collected for all subjects were evaluated together for different possible cut-off values (to distinguish between measured values considered deviant or normal). The area under the ROC (receiver operating characteristics, for an overview over ROC analysis see for instance [21]) curve (AUROC) was used to determine which of the two methods was more suitable for data evaluation. Afterwards, the maximum value of the Youden index [22] was evaluated and used for the selection of the optimum cut-off values. The percentile corresponding to the optimum cut-off values was calculated. 

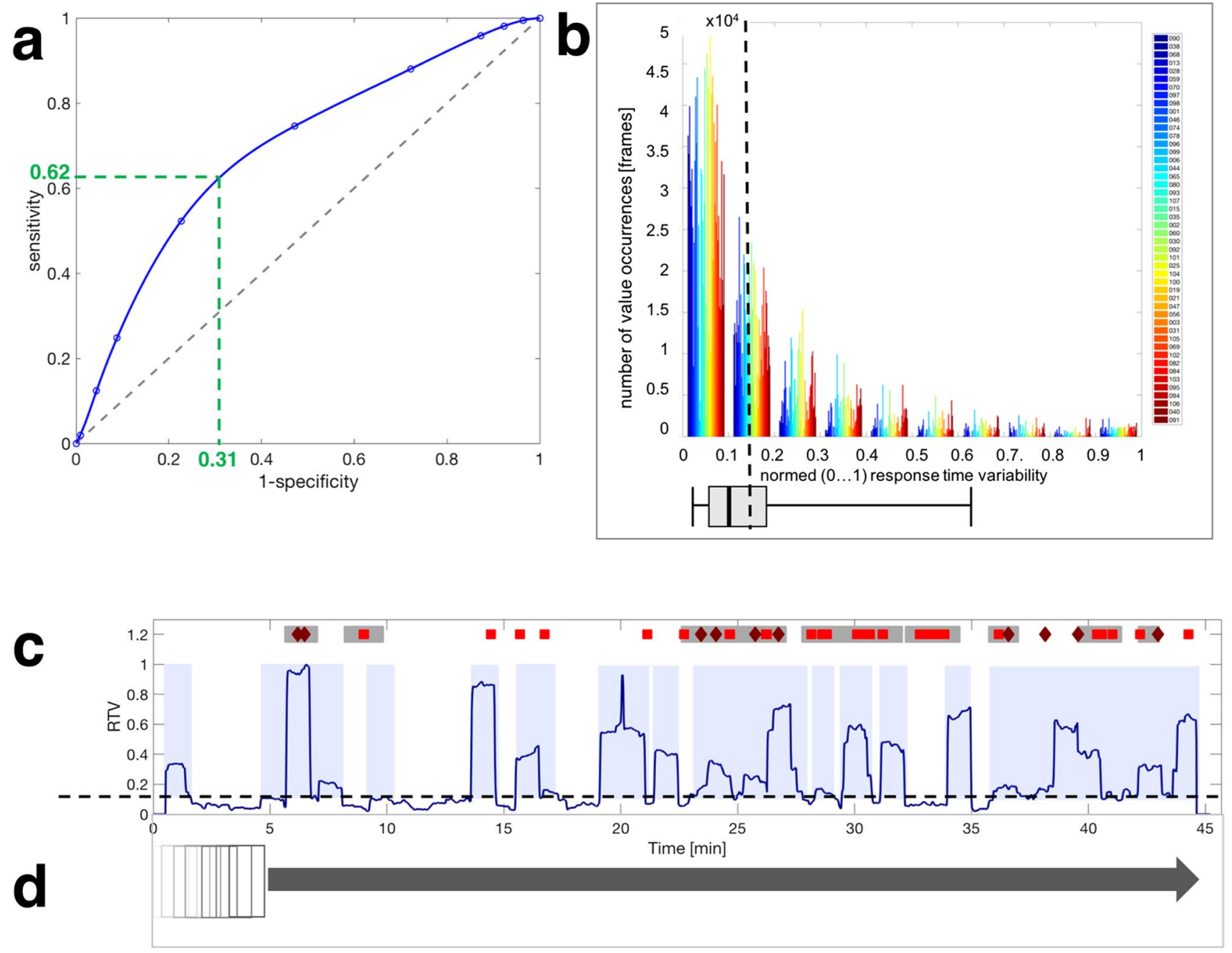

Fig. 1 Example of the global analysis of response time variability (RTV) for subject 106. a The ROC curve for all subjects was calculated and "cut-off" values valid for the entire subject sample for sensitivity and specificity were determined via the Youden index (here: sensitivity $=0.62$ and specificity $=0.69$, see green dotted lines). b Using a distribution function for the RTV for the entire subject sample (the subjects were sorted in ascending order of their total number of errors, color-coded from blue: low number of errors to red: high number of errors), the specific "cut-off" value (as explained in a) corresponding to the 66th percentile was determined (here: 0.15, marked by the dashed black line). The box plot shows the distribution of all measured values for the RTV for the entire subject sample, with the thick black line indicating the median, the box marked 25 th and 75 th percentile and the whiskers representing 5th and 95th

Subsequently, a so-called agreement index (AI) was determined: The agreement between time periods with an increased number of false responses to catch trials (which was considered the gold standard) and time periods with deviant response percentile. c Thus, for each subject, periods of time in which values above 0.15 (marked with the black dotted line) were present were defined as deviant values for the RTV (see light blue highlights). This figure showcases that for subject 106 as an example. Periods with an increased number of false responses to catch trials are highlighted in dark grey. The agreement index (AI) defines periods of time as congruent (and therefore as "agreement") for time intervals with coinciding deviant values for both, the number of false responses to catch trials as well as for the RTV according to the global "cut-off" values evaluated as stated in $\mathbf{a}, \mathbf{b}$. In this case, the AI is 0.24. d Simplified graphical illustration of the method of a "sliding window": Data within a windows of $60 \mathrm{~s}$ are evaluated together. The window "slides" over the complete data set frame by frame

time-based values were set into relation to time periods in which only one of the two criteria was considered pathological. The AI was calculated as follows:

$$
\mathrm{AI}=\frac{\text { increased number of false responses to catch trials AND deviant RT-based values }}{\text { increased number of false responses to catch trials OR deviant RT-based values }}
$$




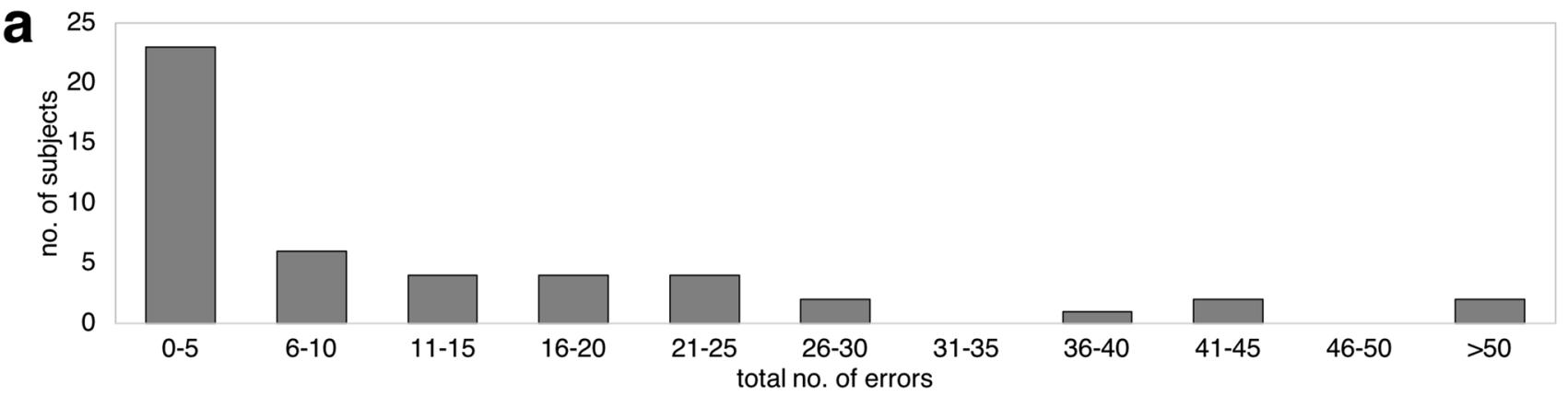

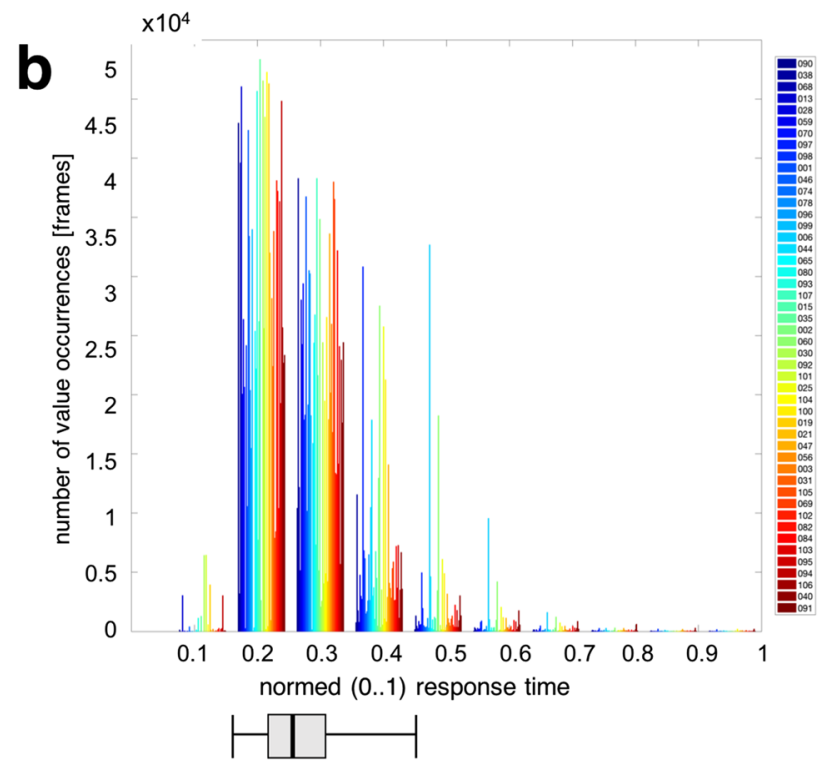

Fig. 2 Frequency distributions of a false responses to catch trials (total number of errors per subject), b normalized (0..1) response time (RT) data (the subjects are shown in ascending order of the total number of errors. A box plot has been added, for which the following applies: box: 25 th and 75 th percentile, black line within the

A representative example for this procedure is shown in Fig. 1.

For individual analysis, the number of false responses to catch trials was calculated "pseudo-continuously" and thus converted into an error rate per minute, which could be correlated to RT and RTV using Spearman rank correlation analysis. Since the correlations were carried out separately for each subject, individual effects could be taken into account, indicating fatigue and/or sleepiness. The evaluation method with the highest median correlation coefficients was selected for the final analysis.

\section{Results}

Each subject was given an ID between 001 and 107 . For organizational reasons, not every possible number was assigned. Fifty-one numbers were assigned to potential subjects, all of whom underwent a preliminary

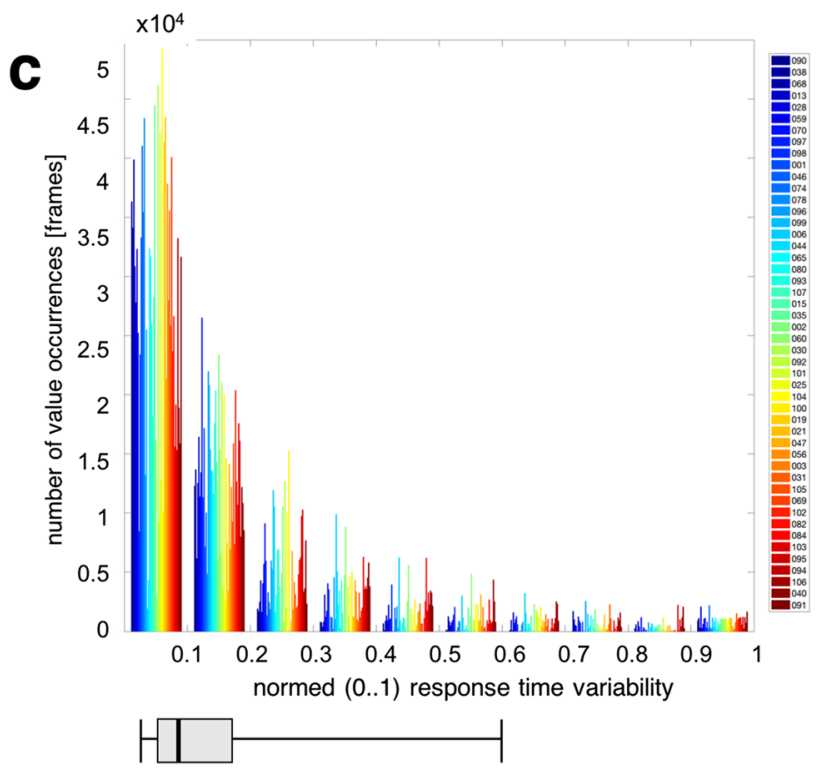

box: median, whisker: 5th and 95th percentile), c normalized (0..1) response time variability (RTV) data (the subjects are shown in ascending order of the total number of errors. A box plot has been added for which the following applies: box: 25 th and 75th percentile, black line inside the box: median, whisker: 5th and 95th percentile)

ophthalmological-optical examination. Two of the test persons examined had to be excluded (reasons: strabismus (1), suspicion of optic nerve disease (1)). One subject ended his participation in the study after the preliminary examination.

Data sets of 48 test persons (18 male, 30 female, age 22-78 years, median age 47 years) were collected and analyzed. In one case (subject 044), the data collection stopped after some time for unknown reasons; the data available to the timepoint were nevertheless analyzed as well.

\section{Parameter distribution}

The frequency distribution of data set was analyzed. False responses to catch trials were considered as the gold standard for the quality of responses in this study (see Fig. 2a). Data were not normally distributed (Shapiro-Wilk test, $W=0.69599, p=1.13 \times 10^{-8}$ ). 
Fig. 3 ROC (receiver operating characteristics) curves and corresponding values for the area under the ROC curve (AUROC) for a response time (RT) and $\mathbf{b}$ response time variability (RTV)
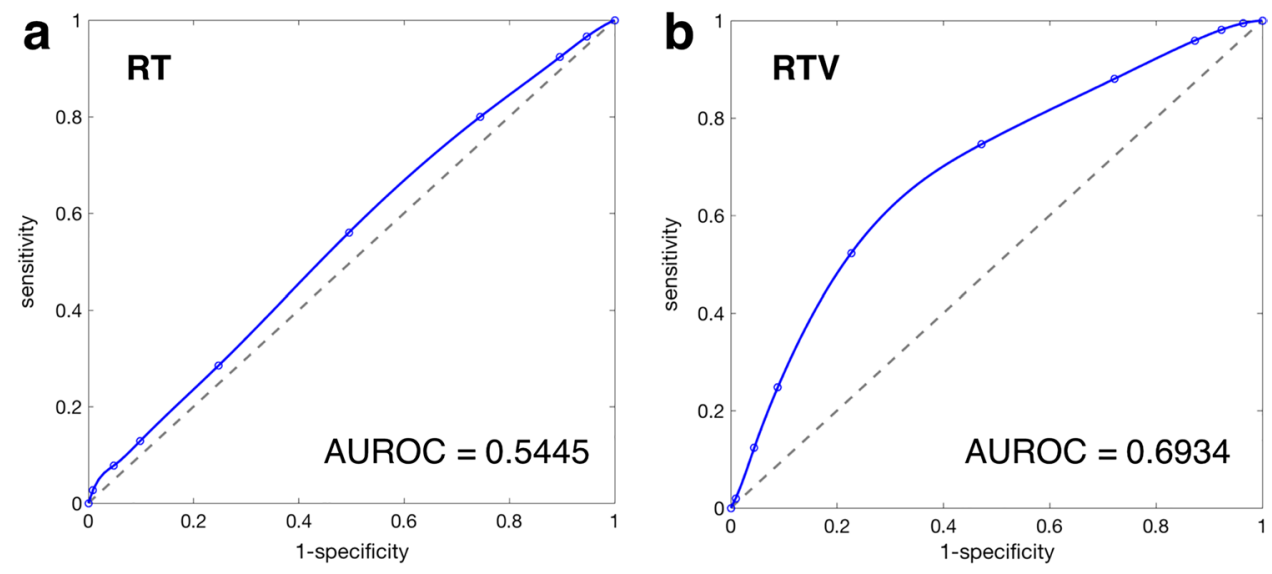

For this reason, non-parametric tests were selected for further evaluation. The median absolute RT for all subjects was $355 \mathrm{~ms}$ with an interquartile range (IQR) of $110 \mathrm{~ms}$. For normalized RT and RTV, histograms were created to show the distribution of individual values for all subjects together (see Fig. 2b, c). As can be derived qualitatively from Fig. 2b, c, RT and RTV are also not normally distributed.

The total number of false responses to catch trials during each of the entire 45-min examinations was (median/ maximum): 6/82. Here and from now on, results are displayed in this manner (median/maximum), as many patients had only a low number of false responses to catch trials. The method presented in this paper shows its strength when there are high numbers of errors-to illustrate this, in addition to the median, no measure of dispersion is given, but rather the maximum.

\section{Global analysis}

The ROC curves for RT and RTV are shown in Fig. 3. Since the AUROC for the RTV (AUROC $=0.6934$ ) was larger than for the RT (AUROC $=0.5445$ ), the RTV appeared to be a more suitable parameter for indicating the response quality during static, automated perimetry.

Cut-off values for sensitivity (0.62) and specificity (0.69) were calculated and corresponded to the 66th percentile of all measured RTV values (as can also be seen in Fig. 1).

Agreement indices (AI) were calculated. Figure 4a shows the AIs of the individual subjects as a function of the total number of errors. The median for those subjects who had periods with an increased number of false responses to catch trials was $\mathrm{AI}_{\text {med }}=0.14$, the maximum value was $\mathrm{AI}_{\max }=0.47$.

Figure 4a shows a "meta-correlation" between AI and the total number of errors. A correlation analysis revealed a Spearman correlation coefficient of $\rho_{\text {global }}=0.91$ $\left(\mathrm{p}=1.06 \times 10^{-19}\right)$.
Fig. 4 a Agreement indices $\left(A I_{R T V}\right)$ for the response time variability (RTV) of the global analysis as a function of the total number of errors $(n)$, red line: linear regression line, $\mathbf{b}$ Individual Spearman correlation coefficients $\left(\rho_{\mathrm{RTV}}\right)$ for the RTV of the individual analysis as a function of the total number of errors $(n)$
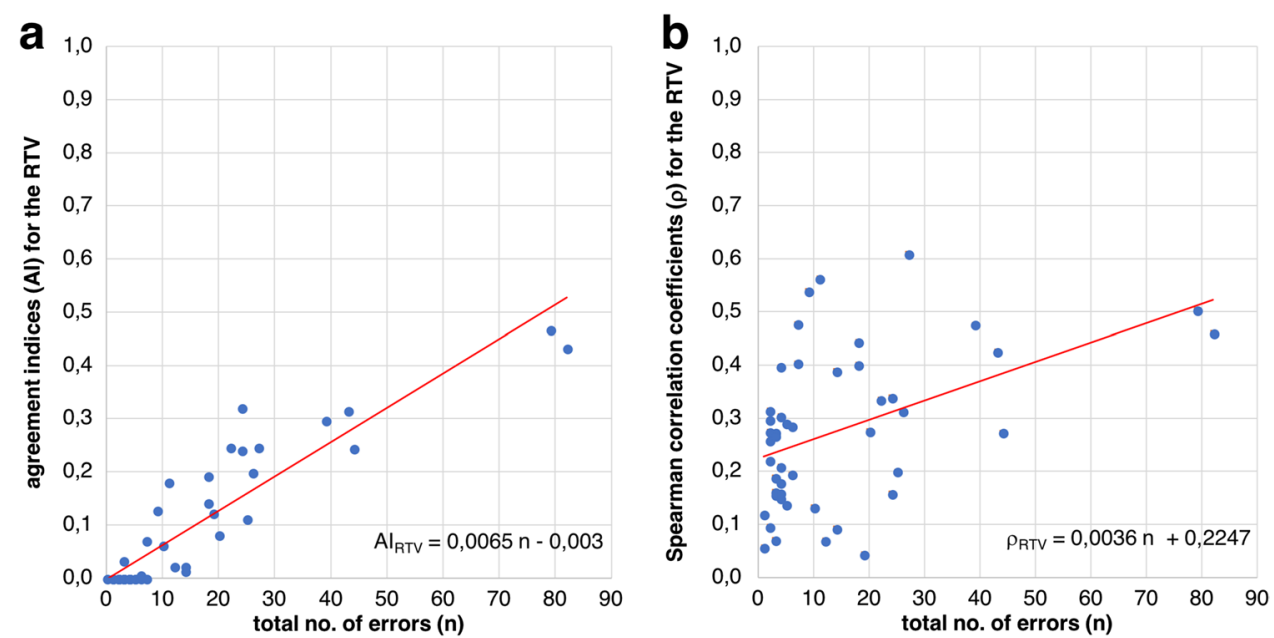


\section{Individual analysis}

Figure $4 \mathrm{~b}$ shows the individual Spearman correlation coefficients as a function of the total number of errors.

Individual Spearman correlation coefficients (median/ maximum) of RT: $\rho_{\mathrm{RT}}=0.05 / 0.35$ and RTV: $\rho_{\mathrm{RTV}}=0.27 / 0.61$ $\left(p_{\max }<10^{-4}\right)$ were obtained. The RTV thus proved to be the more suitable parameter for individual analysis.

In addition, as for the global analysis, a "meta-correlation" was performed between the individual Spearman correlation coefficients and the total number of errors per subject. This resulted in a Spearman correlation coefficient of $\rho_{\text {individual }}=0.45\left(p=1.33 \times 10^{-3}\right)$ and thus a medium, significant correlation.

Similar to the AIs, it was shown that correlations (albeit with lower correlation coefficients) could already be found for subjects with a low total number of errors.

\section{Discussion}

It is known that the circadian rhythm and thus the time of day influence vigilance [10]. For that reason, indicators used to monitor vigilance, also vary during the day [23]. For this reason, the present study tried to distribute the subjects equally over different times of day. For organizational reasons (the examination dates had to be based on the availability of the subjects), however, it was not possible to achieve an equal distribution: All subjects were tested between 08:00 am and 06:00 pm. There was a tendency of testing more subjects in the morning or early afternoon than in the evening. In particular, potential influencing factors such as age or gender could not always be balanced with regard to an equal distribution throughout the day.

In perimetry, rates of 3-5\% for false-positive and falsenegative catch trials are common [6]. This approach thus allows for a "post-hoc" quality control, but its temporal resolution is insufficient to capture the onset of a vigilance incident. Using a rate of $4 \%$, a stimulus duration of $200 \mathrm{~ms}$ and an inter-stimulus interval of $1500 \mathrm{~ms}$, a catch trial is presented approximately every $21 \mathrm{~s}$. Assuming that not every catch trial is necessarily answered incorrectly when fatigue sets in, a rate of $4 \%$ (or even lower) does not seem promising in terms of adequate temporal resolution of fatigue detection. Larger glaucoma or ocular hypertension studies conducted in the past have also used catch trials to test the quality of responses. Some examples are the Advanced Glaucoma Intervention Study (AGIS) [24], the Collaborative Initial Glaucoma Treatment Study (CIGTS) [25] or the Ocular Hypertension Treatment Study (OHTS) [26-28], each with a $3 \%$ catch trial rate. These catch trial rates do not appear to be sufficient for quality control with sufficient temporal resolution. The present study therefore used a rate of $25 \%$ each. The rate implemented in this study leads to a catch trial about every three seconds.

For vigilance assessment during perimetry or campimetry, efforts have been made to monitor or predict errors using pupillography. It has been shown that fatigue waves occur more frequently with increasing examination duration and that the probability of stimulus perception was higher with low amplitudes of pupillary fatigue waves [29]. However, this study was conducted using a small sample size $(n=13)$ of glaucoma patients or suspects with limited age range (51-88 years).

An attempt to predict the error rate of patients via a neural network showed a correlation of $0.72 \pm 0.17$ [30]. However, the correlation coefficients were unstable in comparison between patients, which was shown by the fact that some patients had positive and others negative correlation coefficients. Moreover, only nine structurally similar subjects were included in the study.

Specific questionnaires, such as the Stanford Sleepiness Scale (SSS) [31] and the Epworth Sleepiness Scale (ESS) [32]—with the latter one being the only questionnaire concerning sleepiness being validated in German language to the authors' knowledge-are often used to assess vigilance, which is directly linked to reaction time [18]. Such questionnaires cannot be time-matched but only serve as an "overall" parameter that can be used for an estimation whether vigilance restrictions could occur during an examination in advance or retrospectively. The method presented in this paper is - in contrast-able to induce monotony in a highly standardized manner and to evaluate its effects with high time resolution.

Response times increased with decreasing vigilance. However, the RTV was even more strongly correlated to (decreasing) vigilance than the RT. According to the authors' knowledge, the RTV has not yet been associated with vigilance. Most studies on response time examinations assume that an increased RTV is due to occasional lack of attention [33]. This could be related to a lack of concentration during exhausting tasks such as perimetry.

In contrast to the above-mentioned studies, the present study took response time and its variability into account. Both parameters can be recorded directly or indirectly during perimetry using the OCTOPUS 900 perimeter used (Haag-Streit AG, Koeniz, Switzerland). Only the present study was carried out on a representative (ophthalmologically normal) group of test persons. In direct comparison, the RTV seems to be a comparatively easy parameter to evaluate, which leads to at least comparably good results.

This study has the following weaknesses: Only ophthalmologically normal subjects were included in this study. Although each experiment lasted about $45 \mathrm{~min}$, the subjects experienced considerably fewer periods of sleepiness than initially assumed. This fact had an impact on the data 
analysis: In all cases of missing sleepiness, no correlation with the reaction time-based parameters could be proven. With increasing total number of errors, AI and correlation coefficients between RT/RTV and error rate also increased significantly. Therefore, the method presented in this paper only shows its strength in patients with a high number of false responses to catch trials. However, as patients with an increased number of false responses to catch trials are exactly those who have to be monitored with regard to response quality, this weakness is of relatively low relevance in practice.

Regarding the global analysis of the results, it must be noted that the "cut-off" values were determined empirically using the respective distribution functions.

In summary, an increased number of catch trials was proven as a sufficient validation tool for the assessment of false responses during static perimetry. The RTV turned out as a promising parameter for indicating the quality of responses-both in global and individual terms. The results of the present study indicated that an individual consideration seemed more promising overall.

\section{Outlook}

Patients with advanced visual field defects or neurological diseases could be included in a follow-up study. These patients would generally be expected to have a higher rate of false responses to catch trials $[34,35]$. If such patients were included, the resulting "prevalence enrichment" would probably lead to a stronger correlation for the selected subgroups.

Perimeters that are currently commercially available are capable of recording response times. The RTV could be calculated internally during ongoing perimetry and thus serve as an additional quality parameter, indicating that a continuation of this investigation is not expected to produce psychophysically usable measurement results, but rather represents a vigilance test. For this purpose, an individual baseline would have to be recorded for each patient (e.g. during the first 1-2 min of an examination), to which all other values would then be assessed in relation. As soon as certain limits are exceeded, the examiner is informed to alert the patient or to terminate this perimetric session.

Supplementary Information The online version contains supplementary material available at https://doi.org/10.1007/s00417-021-05349-z.

Funding Open Access funding enabled and organized by Projekt DEAL. This study was financially supported by the Ministry of Science, Research and Arts Baden-Wuerttemberg (Germany) as part of the "HAW-Prom" program.

Data availability The results presented in this paper are part of the dissertation "Assessment of vigilance and response quality during static automated perimetry. A study using the method of constant stimuli (MoCS) and an enhanced presentation rate of catch trials" by Judith
Ungewiss. All data and material are presented and available in this dissertation.

\section{Declarations}

Ethics approval and consent to participate This pilot project was reviewed and approved by the ethics committee of the State Medical Association Baden-Wuerttemberg (Reference No.: F-2018-028). Written informed consent of each participant was obtained prior to the measurements.

Conflict of interest Judith Ungewiss has received a doctoral fellowship with funding from the Ministry of Science, Research and Arts Baden-Wuerttemberg as part of the HAW-Prom program. She received speakers' honorary from AMO Ireland (affiliated to Johnson \& Johnson Vision) and holds patents/patent applications with the numbers 10 2017126 741, WO 2020/089284 A1, and 20174 551.0. Hanspeter A. Mallot reports no conflicts of interest. Ulrich Schiefer is consultant of the Haag-Streit AG, Koeniz/CH and holds several patents concerning psychophysical examination methods, such as visual field testing, glare testing and color vision testing.

Other The content of this paper is part of the dissertation "Assessment of vigilance and response quality during static automated perimetry. A study using the method of constant stimuli (MoCS) and an enhanced presentation rate of catch trials" by Judith Ungewiss. This topic was presented at the DOG 2020 Congress with the title "Die Reaktionszeit und deren Variabilität als Prädiktor der individuellen Antwortqualität während statischer, automatischer Perimetrie".

Open Access This article is licensed under a Creative Commons Attribution 4.0 International License, which permits use, sharing, adaptation, distribution and reproduction in any medium or format, as long as you give appropriate credit to the original author(s) and the source, provide a link to the Creative Commons licence, and indicate if changes were made. The images or other third party material in this article are included in the article's Creative Commons licence, unless indicated otherwise in a credit line to the material. If material is not included in the article's Creative Commons licence and your intended use is not permitted by statutory regulation or exceeds the permitted use, you will need to obtain permission directly from the copyright holder. To view a copy of this licence, visit http://creativecommons.org/licenses/by/4.0/.

\section{References}

1. Traquair HM (1938) An introduction to clinical perimetry, Chpt. 1. Henry Kimpton, London, pp 4-5

2. Heijl A, Drance SM, Douglas GR (1980) Automatic perimetry (COMPETER). Ability to detect early glaucomatous field defects. Arch Ophthalmol 98:1560-1563

3. Heijl A, Krakau CET (1975) An automatic perimeter for glaucoma visual field screening and control: construction and clinical cases. Albrecht von Graefes Arch Klin Ophthalmol 197:13-23. https:// doi.org/10.1007/BF00506636

4. Anderson DR (1987) Perimetry: with and without automation. CRC Press, Cambridge

5. Demirel S, Vingrys A (1995) Eye movements during perimetry and the effect that fixational instability has on perimetric outcomes. Ophthalmic Lit 2:145

6. Schiefer U, Pätzold J, Wabbels B, Dannheim F (2006) Konventionelle Perimetrie - Teil 4: Statische Perimetrie: 
Befundauswertung - Indizes - Verlaufskontrolle - Perimetrie im Kindesalter. Ophthalmologe 103:235-254

7. Johnson CA, Adams CW, Lewis RA (1988) Fatigue effects in automated perimetry. Appl Opt 27:1030-1037

8. Canisius S, Penzel T (2007) Vigilance monitoring-review and practical aspects. Biomed Tech 52:77-82

9. Head H (1923) The conception of nervous and mental energy (II). Br J Psychol 14:126

10. Wilhelm B, Giedke H, Lüdtke $H$ et al (2001) Daytime variations in central nervous system activation measured by a pupillographic sleepiness test. J Sleep Res 10:1-7

11. Weeß H, Lund R, Gresele C et al (1998) Vigilanz, Einschlafneigung, Daueraufmerksamkeit, Müdigkeit, Schläfrigkeit. Somnologie-Schlafforschung und Schlafmedizin 2:32-41

12. Chaudhuri A, Behan PO (2004) Fatigue in neurological disorders. The Lancet 363:978-988

13. Weeß H, Sauter C, Geisler P et al (2000) Vigilanz, Einschlafneigung, Daueraufmerksamkeit, Müdigkeit, Schläfrigkeit. Diagnostische Instrumentarien zur Messung müdigkeits- und schläfrigkeitsbezogener Prozesse und deren Gütekriterien: vigilance, tendency to fall asleep, sustained attention, tiredness, sleepiness diagnostic tools for the measurement of sleepiness related processes and their criteria of quality. Somnologie 4:20-38

14. Sunga RN, Enoch JM (1970) Further perimetric analysis of patients with lesions of the visual pathways. Am J Ophthalmol 70:403-422. https://doi.org/10.1016/0002-9394(70)90101-7

15. Enoch JM, Berger R, Birns R (1970) A static perimetric technique believed to test receptive field properties: extension and verification of the analysis. Doc Ophthalmol 29:127-153

16. Wall M, Maw RJ, Stanek KE, Chauhan BC (1996) The psychometric function and reaction times of automated perimetry in normal and abnormal areas of the visual field in patients with glaucoma. Invest Ophthalmol Vis Sci 37:878-885

17. Wall M, Kutzko KE, Chauhan BC (2002) The relationship of visual threshold and reaction time to visual field eccentricity with conventional automated perimetry. Vision Res 42:781787. https://doi.org/10.1016/S0042-6989(01)00311-X

18. Surwillo WW, Quilter RE (1964) Vigilance, age, and responsetime. Am J Psychol 77:614-620

19. Urban FM (1910) The method of constant stimuli and its generalizations. Psychol Rev 17(4):229-259

20. Turpin A, Artes PH, McKendrick AM (2012) The open perimetry interface: an enabling tool for clinical visual psychophysics. J Vis. https://doi.org/10.1167/12.11.22

21. Fawcett T (2006) An introduction to ROC analysis. Pattern Recogn Lett 27(8):861-874
22. Youden WJ (1950) Index for rating diagnostic tests. Cancer 3:32-35

23. Kraemer S, Danker-Hopfe H, Dorn H et al (2000) Time-of-day variations of indicators of attention: performance, physiologic parameters, and self-assessment of sleepiness. Biol Psychiat 48:1069-1080

24. The AGIS Investigators (2000) The Advanced Glaucoma Intervention Study (AGIS): 7. The relationship between control of intraocular pressure and visual field deterioration. Am J Ophthalmol 130:429-440

25. Wahl J (2005) Ergebnisse der „Collaborative Initial Glaucoma Treatment Study (CIGTS). Ophthalmologe 102:222-226

26. Johnson CA, Keltner JL, Cello KE et al (2002) Baseline visual field characteristics in the ocular hypertension treatment study. Ophthalmology 109:432-437

27. Kass MA, Heuer DK, Higginbotham EJ et al (2002) The Ocular Hypertension Treatment Study: a randomized trial determines that topical ocular hypotensive medication delays or prevents the onset of primary open-angle glaucoma. Arch Ophthalmol 120:701-713

28. Keltner JL, Johnson CA, Cello KE et al (2003) Classification of visual field abnormalities in the ocular hypertension treatment study. Arch Ophthalmol 121:643-650

29. Henson DB, Emuh T (2010) Monitoring vigilance during perimetry by using pupillography. Invest Ophthalmol Vis Sci 51:3540-3543

30. Vergani Dambros G, Ungewiss J, Kübler T, Kasneci E, Spüler M (2017) Monitoring response quality during campimetry via eyetracking. In: Proceedings of the 22nd International Conference on Intelligent User Interfaces Companion, pp 61-64

31. Hoddes E, Zarcone V, Smythe H, Phillips R, Dement WC (1973) Quantification of sleepiness: a new approach. Psychophysiology 10(4):431-436

32. Johns MW (1991) A new method for measuring daytime sleepiness: the Epworth Sleepiness Scale. Sleep 14(6):540-545

33. Tamm L, Narad ME, Antonini TN et al (2012) Reaction time variability in ADHD: a review. Neurotherapeutics 9:500-508

34. Birt CM, Shin DH, Samudrala V et al (1997) Analysis of reliability indices from Humphrey visual field tests in an urban glaucoma population. Ophthalmology 104:1126-1130

35. Hom J, Reitan RM (1990) Generalized cognitive function after stroke. J Clin Exp Neuropsychol 12:644-654

Publisher's note Springer Nature remains neutral with regard to jurisdictional claims in published maps and institutional affiliations. 